\title{
Aspectos que influyen en la falta de utilización de los servicios de un programa de esteriliza - ción.
}

\author{
* Dr.: Néstor H. Amorocho P. \\ ** Martha Lucía Quintero H. \\ *** Beatríz Eugenia González \\ **** Luz Stella Olivo
}

\section{INTRODUCCION}

La esterilización ha sido muy utilizada en el Oriente y en los Estados Unidos. Entre nosotros se inició en 1970 con un programa de Vasectomía y en 1973 con la Esterilización Voluntaria Femenina con las nuevas Técnicas de Laparoscopia y Minilaparatomía. Puesto que el objetivo principal de este trabajo es determinar los factores que inciden en el no uso de la Esterilización, revisamos las estadísticas de 1977, deteniéndonos particularmente en el segundo semestre del mismo año. Se observa en el cuadro y en la gráfica No. 1 un aumento progresivo en los meses de Junio, Julio y Agosto para luego declinar progresivamente en los últimos meses del semestre, sin existir una razón explícita que justifique tal hecho.

En consideración a lo anterior y con miras a investigar el hecho observado se pensó, que por medio de una.entrevista personal a un grupo de señoras se llegara a detectar las causas por las cuales algunas de ellas no acuden al programa estando en condiciones de hacerlo y otras que se retractan después de iniciadas las gestiones necesarias para ser admitidas en él.

Para aprovechar al máximo la encuesta se agregaron preguntas, complementándolas con miras a obtener resultados que permi-

* Jefe Depto. de Cirugía

Depto. Educativo

** Instructora Capacitación Institucional

$* * *$ Encuestadora

**** Instructora Programa Comunitario Profamilia - Centro de Cali - Enero 1978. tan el mejoramiento de los programas institucionales de acuerdo al concepto que como Institución Educativa tuviese la Entidad en el grupo materia de estudio. (Anexo No. 1).

El Departamento de Información, Educación, Comunicación (I.E.C.) está a cargo de Instructoras Móviles e Instructoras de Centro. Las primeras llevan la información y educación de Planificación Familiar a la comunidad y colaboran con el Programa Comunitario Urbano, Puestos de Distribución, Información y Divulgación sobre los servicios de Planificación Familiar lo que facilita el acceso popular a los métodos anticonceptivos, estableciendo de esta manera un sistema de remisión hacia el Centro.

Las Instructoras de Centro orientan a las potenciales usuarias, acompañantes y público en general, que llegan a la Sede.

La orientación está dirigida hacia los diferentes métodos anticonceptivos informando sobre uso, beneficios, efectos colaterales y la importancia del uso continuado de un método cualquiera, escogido libremente por la persona.

La labor llevada a cabo en el Segundo Semestre de 1977 en términos generales puede resumirse así:

En el Programa Comunitario se dictaron 22 charlas sobre Planificación Familiar con una asistencia global de 664 personas.

34 Charlas de Capacitación a Distribuidores.

A nivel de Empresas e Instituciones se visitaron 60 Empresas, dictados 22 cursillos 


\section{ASOCIACION PRO-BIENESTAR DE LA FAMILIA COLOMBIANA}

\section{A L I}

1) NOMBRE EDAD AÑNOS

2) DIRECCION

3) OCUPACION

4) NUMERO DE HIJOS

5) OCUPACION DEL ESPOSO

6) INGRESOS MENSUALES ENTRE:
a) MENOS DE
1.000 .00
b) 1.000 .00
- 2.000 .00
c) 2.000 .00
- 3.000 .00
d) 3.000 .00
- 4.000 .00
e) 4.000.0o
- 5.000 .00
f) MAS DE
5.000 .00

7) QUE ENTIENDE USTED POR PLANIFICACION FAMILIAR?

8) COMO OBTUVO ESTA INFORMACION?

9) QUE METODOS CONOCIA ANTES DE IR A PROFAMILIA?

10) CUANTO HACE QUE FUE AL CENTRO POR PRIMERA VEZ?

11) EN QUE SITIO DEL CENTRO RECIBIO LA PRIMERA INFORMACION?
a) Portería
e) Consultorios
b) Recepción
f) Otros
c) Caja
d) Sala de Conferencias

12) SOLICITO USTED MAS INFORMACION?

SI

NO

13) EN QUE OTRO SITIO DEL CENTRO?

14) QUE METODOS DF, PLANIFICACION LE ENSEÑARON?
a) Ritmo
e) Tabletas
b) Pildoras
f) Ovulos
c) Diu
g) Espuma
d) Condón
h) Cirugía 
15) ENTRE LOS METODOS QUE CONOCIO CUAL ESCOGIO?

NOTA: En caso de que no mencione cirugía dar explicación al respecto y hacer las preguntas Ns. $16-20-21$.

NOTA: Si la señora responde cirugía seguir con las preguntas Ns. 17 y 18.

16) QUE OPINA USTED SOBRE EL PROGRAMA?

17) QUE REQUISITOS LE EXIGIERON?

18) SE LA EFECTUARON?

SI DONDE?

No

19) POR QUE?

(Hacer preguntas 19-20-21

a) No tuvo con quien dejar los niños?

b) El esposo se arrepintió?

c) No tuvo dinero para el transporte?

d) Tuvo miedo de la operación?

e) Cree que la operación afecta las relaciones sexuales

f) Duda de la seguridad del método?

g) Estaba muy reciente el parto

h) Motivos religiosos

i) Otros

20) ACTUALMENTE UTILIZA ALGUN METODO DE PLANIFICACION? CUAL? ESTA USTED INSCRITA A PROFAMILIA?

ENCUESTADA

ENCUESTADORA

Cali, de 1.97

completos, y 141 Conferencias.

Quedó organizada la motivación diaria extra-mural previa capacitación y orientación de una persona apta para ella en Instituciones como: Cruz Roja, Policía Nacional, etc.

Del Centro se obtuvieron los siguientes datos: 6 Charlas diarias de aproximadamente 30 minutos de duración por cada una, sobre todos los métodos de Planificación, con un promedio de 370 personas motivadas mensualmente.

\section{OBJETIVOS}

1. Determinar los factores que influyen en el no uso del programa de esterilización.

2. Proponer sugerencias para el mejoramiento de los programas de la Institución.

3 Averiguar la imagen de Profamilia como Instituciīn Educativa y de ayuda a la comunidad en cuanto a aspectos de Planificación Familiar se refiere. 


\begin{tabular}{|l|r|r|r|r|r|}
\hline \multicolumn{6}{|c|}{$\begin{array}{r}\text { CUADRO ESTADISTICO SOBRE EL PROGRAMA QUIRUR GICO } \\
\text { SEGUNDO SEMESTRE 1977 }\end{array}$} \\
\hline MESES & T.OPERACIONES & T. FALLAS $\%$ & FALLAS ISS & FALLAS CENTRO \\
\hline JUNIO & 231 & 37 & 15.61 & 28 & 9 \\
\hline JULIO & 263 & 44 & 18.56 & 32 & 12 \\
\hline AGOSTO & 322 & 57 & 24.10 & 38 & 19 \\
\hline SEPTIEMBRE & 283 & 40 & 16.87 & 25 & 15 \\
\hline OCTUBRE & 251 & 42 & 17.72 & 34 & 8 \\
\hline NOVIEMBRE & 118 & 17 & 7.17 & 10 & 7 \\
\hline TOTALES & 1.468 & 237 & 100.0 & 167 & 70 \\
\hline & - & $16.14(16)$ & - & $11.37(11)$ & $4.76(4)$ \\
\hline
\end{tabular}

\section{CUADRO No. 1}

\section{METODOLOGIA}

La Metodología empleada fué la entrevista personal con la aplicación del cuestionario anexo No. 1. se determinó el número de la muestra por medio de la técnica "Muestra al azar sin reemplazamientos" para que esta fuera representativa de la población se tomó el $80 \%$ así: De las 237 fallas entrể Junio y Noviembre 15 de 1977 el $80 \%$ corresponde a 189.6 lo que es igual a 190 historias. aparecen $13 \sin$ dirección.

1. Caso Especial. (Aplazada la intervención quirúrgica hasta definir situación obstétrica, Es de anotar que dicha señora volvió al Centro y fué operada).

2. Fuera de la ciudad así:

10 Yumbo

\section{Florida}

\section{Veredas}

3 Jamundí

Debido a la limitación del tiempo no se contó con las señoras que vienen fuera de la ciudad, en total quedó así:

$$
190-3^{*}=155
$$

Se encontraron los siguientes inconvenientes

40 Dirección errada

23 No se encontraron en el momento de la visita

12 Dirección que no corresponde

4 Señoras que han cambiado de casa r.

Quedando reducido a 76 encuestas correspondientes al $40 \%$ realizadas por tres encuestadoras, las cuales gastaron un tiempo total de 52,20 (Cincuenta horas, $20 \mathrm{minu}$ tos) y cuyo costo fue de $\$ 84.00$ por hora de encuesta.

Para efectos estadísticos se utilizó la fórmula: $x$ EFPM (Promedio de la suma de la frecuencia por punto medio sobre número de datos)

Para hallar el promedio como medida representativa del conjunto de datos. 
X: Promedio: Valor representativo de conjunto de datos.

E: Sumattorias: Símbolo indicativo de suma.

F: Frecuencia: Número de veces que se repite un dato.

M: Punto medio

N: Número de datos: Total de entrevistas realizadas.

ANALISIS DE DATOS

EDAD POR GRUPOS

\begin{tabular}{|c|c|c|c|c|}
\hline EDADES & F & $\%$ & PM & F.P.M \\
\hline $25-26$ & 12 & 15.7 & 24.5 & 294 \\
\hline $27-31$ & 29 & 38.15 & 29.5 & 855.5 \\
\hline $32-36$ & 21 & 27.6 & 34.5 & 724.5 \\
\hline $37-41$ & 11 & 14.47 & 39.5 & 434.5 \\
\hline $42-46$ & 3 & 3.94 & 44.5 & 133.5 \\
\hline Totales & 76 & 100. & - & 2.442 .0 \\
\hline
\end{tabular}

CUADRO No. 2

En el cuadro No. 2 aparecen las pacientes encuestadas por grupos de edad. La menor correspondió a 22 años y la mayor a 45 años. El mayor porcentaje fue de $38.15 \%$ para el grupo comprendido entre 27 y 31 años; la edad promedio fue de 32 años.

OCUPACION DE LA USUARIA

\begin{tabular}{|l|c|r|}
\hline OCUPACION & No. & $\%$ \\
\hline Hogar & 64 & 84.2 \\
\hline Operarias & 7 & 9.21 \\
\hline Auxiliares Enfermería & 3 & 3.94 \\
\hline Profesional & 1 & 1.32 \\
\hline Magisterio & 1 & 1.32 \\
\hline TOTALES & 76 & 100 \\
\hline
\end{tabular}

CUADRO No. 3
El cuadro No. 3 representa la ocupación de las señoras, de las cuales el $84.2 \%$ cataloga a las amas de casa y el resto a otras ocupaciones:

Operarias $(9.2 \%)$, profesionales $(1.32 \%)$

NUMERO DE HIJOS VIVOS

\begin{tabular}{|l|c|c|c|c|c|}
\hline No. & Hijos & $\mathrm{F}$ & $\%$ & PM & F.P.M. \\
\hline 0 & -2 & 17 & 22.3 & 1.5 & 22.5 \\
\hline 3 & -5 & 46 & 60.6 & 4.5 & 207 \\
\hline 6 & -8 & 12 & 15.8 & 7.5 & 90.0 \\
\hline 9 & -11 & 1 & 1.3 & 10.5 & 10.5 \\
\hline \multicolumn{5}{|l}{ TOTALES $76100-333.0$} \\
\hline
\end{tabular}

Cuadro No. 4

El número de hijos oscila entre 1 y 10 obteniéndose el mayor porcentaje 60.6 para el grupo de clase comprendido entre 3 y 5. El promedio resultó ser de 4 hijos. (Gráfica No. 2).

CCUPACION DEL ESPOSO

\begin{tabular}{|l|c|r|}
\hline OCUPACION & F & $\%$ \\
\hline Obrero & 35 & 46.05 \\
\hline Empleado & 21 & 27.63 \\
\hline Comerciante & 11 & 14.48 \\
\hline Profesional & 6 & 7.89 \\
\hline Otros & 3 & 3.95 \\
\hline TOTALES & 76 & 100 \\
\hline
\end{tabular}

CUADRO No. 5

En cuanto a la ocupación del esposo -cuadro No. 5- el mayor porcentaje corresponde a la categoría "obrero,, siguiendo en su orden: Empleado, Comerciante y Profesional. En la clase "otros" están catalogados los que no tienen ocupación. 
INGRESO MENSUAL FAMILIAR

\begin{tabular}{|l|c|r|}
\hline INGRESOS & F & \multicolumn{1}{|c|}{$\%$} \\
\hline Menos de 1.000 & 0 & - \\
\hline $1.001-2.000$ & 15 & 19.73 \\
\hline $2.001-3.000$ & 19 & 25.3 \\
\hline $3.001-4.000$ & 18 & 23.68 \\
\hline $4.001-5.000$ & 12 & 15.78 \\
\hline Más de 5.000 & 12 & 15.78 \\
\hline TOTALES & 76 & 100 \\
\hline
\end{tabular}

CUADRO No. 6

Según el cuadro No. 6 . el $49 \%$ de estas familias tienen un ingreso mensual familiar que va entre $2.001 \mathrm{y} \operatorname{los} 4.000$ pesos

CONOCIMIENTO SOBRE PLANIFICACION FAMILIAR

\begin{tabular}{|l|l|r|}
\hline CONCEPTOS & F & \multicolumn{1}{|c|}{$\%$} \\
\hline Evitar los hijos & 44 & 57.89 \\
\hline Métodos para regular & & \\
\hline la familia & 5 & 6.57 \\
\hline $\begin{array}{l}\text { Tener los hijos que se } \\
\text { puedan educar y man } \\
\text { tener }\end{array}$ & & \\
\hline $\begin{array}{l}\text { Bienestar para la } \\
\text { familia }\end{array}$ & 3 & 27.63 \\
\hline $\begin{array}{l}\text { Control de la Nata- } \\
\text { lidad }\end{array}$ & 1 & 3.95 \\
\hline No tener familia & 1 & 1.32 \\
\hline No sabe & 1 & 1.32 \\
\hline TOTALES & 76 & 100 \\
\hline
\end{tabular}

\section{CUADRO No. 7}

El conocimiento sobre Planificación Familiar está basado fundamentalmente en el concepto: "Evitar la Familia" obteniéndose esta respuesta de 44 señoras correspondientes a $57.89 \%$.
FUENTES DE INFORMACION

DE LA USUARIA

\begin{tabular}{|l|c|r|}
\hline LUGAR & F & \multicolumn{1}{|c|}{$2 \%$} \\
\hline Centro de Salud & 20 & 26.32 \\
\hline $\begin{array}{l}\text { Instituto Seguros } \\
\text { Sociales (profami- } \\
\text { lia) }\end{array}$ & 16 & 21.05 \\
\hline Centro Profamilia & 14 & 18.42 \\
\hline Amigas & 8 & 10.53 \\
\hline Radio & 5 & 6.58 \\
\hline Médico Particular & 4 & 5.26 \\
\hline Conferencias & 3 & 3.95 \\
\hline Experiencia de la vida & 4 & 5.26 \\
\hline Ninguno & 2 & 2.63 \\
\hline TOTALES & 76 & 100 \\
\hline
\end{tabular}

\section{CUADRO No. 8}

La mayor fuente de información procede de los Centros de Salud, en segundo orden el Seguro Social (ISS) y en un tercer orden Profamilia. Es de anotar que algunas de las señoras no han obtenido una información especifica, manifiestan que la han adquirido por experiencia propia y la realidad de la vida.

\section{METODOS QUE CONOCIA ANTES DE IR A PROFAMILIA}

Dado que esta pregunta es de tipo abierta, resultaron muchas combinaciones de métodos conocidos. Para mayor facilidad se tomó el siguiente criterio:

1.- El método que aparecía con màyon frecuencia en todos los grupos preliminares.

2.- Los métodos que aparecían solos.

Resultaron así: 


\begin{tabular}{|l|c|r|}
\hline METODOS & $F$ & $\%$ \\
\hline Píldora & 41 & 53.94 \\
\hline No conoce & 30 & 39.47 \\
\hline D.I.U. & 2 & 2.63 \\
\hline Ritmo & 2 & 2.63 \\
\hline Ovulos & 1 & 1.31 \\
\hline TOTALES & 76 & 100 \\
\hline
\end{tabular}

\section{CUADRO No. 9}

El método más conocido es la "píldora" en razón a su alto porcentaje $(53.94 \%)$, un $39.47 \%$ no conoce ningún método; en tercer orden aparece el D.I.U. y el Ritmo con igual porcentaje $(2.63 \%)$, por último los ovulos con $1.31 \%$.

\section{TIEMPO TRANSCURRIDO DESDE LA PRIMERA VISITA A PROFAMILIA}

\begin{tabular}{|l|r|}
\hline TIEMPO EN MESES & F \\
\hline $0-13$ & 29 \\
\hline $14-27$ & 2 \\
\hline $28-41$ & 7 \\
\hline $42-55$ & 3 \\
\hline $56-69$ & 5 \\
\hline $70-83$ & 7 \\
\hline $84-97$ & 5 \\
\hline TOTALES & 58 \\
\hline
\end{tabular}

CUADRO No. 10

El tiempo transcurrido desde cuando vino por primera vez a Profamilia hasta el momento de la encuesta varía de 1 mes a 8 años -94 meses - para efectos de tabulación se tomó el tiempo en meses, observándose una mayor frecuencia 29 usuarias correspondientes al grupo de 0-13 meses.

Es de hacer notar que 18 señoras fueron entrevistadas en el ISS fueron remitidas al Centro directamente al Programa Quirúrgico por la Instructora de Profamilia.

\section{SITIO DEL CENTRO DONDE RECIBIO}

\section{LA PRIMERA INFORMACION}

\begin{tabular}{|l|c|r|}
\hline SITIO & F & \\
\hline Portería & 0 & 0 \\
\hline Recepción & 3 & 3.94 \\
\hline Caja & 5 & 6.57 \\
\hline $\begin{array}{l}\text { Sala de Conferen- } \\
\text { cias }\end{array}$ & 22 & 18.94 \\
\hline Consultorios & 0 & 0 \\
\hline Otros (ISS) & 46 & 60.52 \\
\hline TOTALES & 76 & 100 \\
\hline
\end{tabular}

\section{CUADRO No. 11}

En el cuadro No. 11 se observa que el sitio del Centro donde las señoras recibieron la primera información corresponde a la Sala de Conferencias. Un pequeño porcentaje recibe la información en otro sitio del Centro, el resto de señoras han sido motivadas en el ISS.

\section{SOLICITO MAS INFORMACION}

Excluyendo las 46 del Seguro, de las 30 motivadas en el Centro $18(60 \%)$ si solicitan más información, solo $12(40 \%)$ no lo hicieron.

\section{EN QUE SITIO DEL CENTRO}

De las 18 que SI solicitaron más información:

15- No contestaron en qué sitio.

3- En la sala de Conferencias. 
METODOS DE PLANIFICACION FAMILIAR QUE LE ENSEÑARON

\begin{tabular}{|l|c|c|c|c|}
\hline \multicolumn{4}{|l|}{ METODOS CENTPO I.S.S. TOTAL } \\
\hline Sólo 1 método & 2 & 20 (Cirugía) & 22 & 28.94 \\
\hline 2-3 métodos & 2 & 10 & 12 & 15.78 \\
\hline 4-5 métodos & 6 & 12 & 18 & 23.98 \\
\hline 6-7 métodos & 18 & 4 & 22 & 28.94 \\
\hline $\begin{array}{l}\text { Todos los mé- } \\
\text { todos }\end{array}$ & 2 & 0 & 2 & 2.63 \\
\hline TOTALES & 30 & 46 & 76 & 100 \\
\hline
\end{tabular}

\section{CUADRO No. 12}

Según el cuadro No, 12. Las señoras demuestran captar más la información que reciben sobre Píldoras y D.I.U., que corresponden a la clasificación entre 4 y 7 métodos, incluídos éstos.

E1 $28.94 \%$ recibió información sobre la mayoría de los métodos ((6-7), otro porcentaje igual sobre un solo método: Cirugía, correspondiente en su mayor parte a las señoras procedentes del ISS.

\section{METODO ESCOGIDO}

De las 46 señoras motivadas en el ISS, 9 en primera instancia escogieron otro método: 5 D.I.U. -3 Pildoras- 1 Ritmo que luego cambiaron a Cirugía.

De las 30 señoras motivadas en el Centro, 3 en piimera instancia escogieron otro método: 1 el D.I.U. 1 Píldora -1 Ovulos que luego cambiaron a Cirugía.

\section{OPINION SOBRE EL PROGR AMA}

E1 $71 \%$ de las señoras encuestadas manifiestan aceptación del Programa de Cirugía, las otras aunque no demuestran negatividad absoluta, añaden diversos comentarios al respecto, entre éstos:
Muy bueno, pero es riesgozo

Muy bueno, pero a mi esposo no le gusta.

Muy bueno, es seguro

Muy bueno, se evitan los otros métodos

CONOCIMIENTO DE LOS REQUISITOS EXIGIDOS POR PROF AMILIA

\begin{tabular}{|l|c|r|}
\hline $\begin{array}{l}\text { Autorización del } \\
\text { esposo }\end{array}$ & 54 & 71.1 \\
\hline $\begin{array}{l}\text { Exámenes de } \\
\text { laboratorio }\end{array}$ & 9 & 11.84 \\
\hline Ninguno & 9 & 11.84 \\
\hline $\begin{array}{l}\text { Costo de la opera- } \\
\text { ción }\end{array}$ & 4 & 5.27 \\
\hline TOTALES & 76 & 100 \\
\hline
\end{tabular}

CUADRO no. 13

Entre los requisitos exigidos para la realización de las Cirugías, Cuadro No. 13. Las encuestadas sabían sobre la autorización firmada por el Esposo: $71.1 \%$, no conocian ningún requisito: $11.84 \%$

\section{SE EFECTUO LA INTERVENCION}

De las 76 encuestadas se encontraron 8 señoras ya operadas asi, 7 en Profamilia (1 vasectomía para el esposo) 1 en Hospital Deptal. 68 sin efectuarse la Operación.

De las 68 operaciones no efectuadas aún, el $29 \%$ aduce como razón principal: "el miedo a la operación" como segunda causa principal aparece "arrepentimiento del esposo", un $19.11 \%$ manifiestan como razón "otros", motivos que aparecen a continuación. Gráfica No. 3 Enfermedad - 6 (artritis, bronquitis, encefalitis)

Sospecha de embarazo -1 


\section{RAZONES DE NO ASISTENCIA A LA INTERVENCION}

\begin{tabular}{|l|c|r|}
\hline RAZONES & F & $\%$ \\
\hline $\begin{array}{l}\text { Tuvo miedo de la } \\
\text { operación }\end{array}$ & 20 & 29.41 \\
\hline $\begin{array}{l}\text { Esposo se } \\
\text { arrepintió }\end{array}$ & 18 & 26.47 \\
\hline Otros & 13 & 19.11 \\
\hline $\begin{array}{l}\text { No tuvo con quien } \\
\text { dejar los niños }\end{array}$ & 8 & 11.76 \\
\hline $\begin{array}{l}\text { Estaba muy } \\
\text { recierte el parto }\end{array}$ & 8 & 1.49 \\
\hline $\begin{array}{l}\text { Cree que la opera- } \\
\text { ción afecta las rela- } \\
\text { ciones sexuales }\end{array}$ & 1 & 100 \\
\hline TOTALES & 76 & \\
\hline
\end{tabular}

CUADRO no. 14

Vacaciones del Esposo - 1

La operación ocasiona enfermedades (hemorragias, Jaquecas, etc.)

Embarazo Positivo - 1

Muy joven (28 años)

Por descuido, olvido $-1^{\text {. }}$

Falta de Dinero - 1

Siendo este item (razones de no asistencia al Programa) uno de los más importantes de la Encuesta, no bastaba con tener una información del grupo en general; por lo tanto se clasificó en dos sub-grupos.

GRUPO A: Señoras motivadas en el ISS

B: Señoras motivadas en el Centro.
I. S. S.

\begin{tabular}{|l|c|r|}
\hline RAZONES & F. & $\%$ \\
\hline $\begin{array}{l}\text { Tuvo miedo a la } \\
\text { Operación }\end{array}$ & 13 & 31.70 \\
\hline $\begin{array}{l}\text { El esposo se } \\
\text { arrepintió }\end{array}$ & 9 & 21.95 \\
\hline $\begin{array}{l}\text { Parto muy } \\
\text { reciente }\end{array}$ & 8 & 19.53 \\
\hline $\begin{array}{l}\text { Otros } \\
\text { No tuvo con } \\
\text { quien dejar los } \\
\text { niños }\end{array}$ & 7 & 17.07 \\
\hline TOTALES & 4 & $\begin{array}{r}9.75 \\
\text { Tros }\end{array}$ \\
\hline \multicolumn{2}{|c|}{$\begin{array}{l}41 \text { no operadas } \\
\text { motivadas en el } \\
\text { ISS }\end{array}$} \\
\hline
\end{tabular}

CUADRO No. 15

En el cuadro No. 15 se encontró nuevamente como razón principal para no efectuarse la operación el "miedo a ella" manifestado por el $31.70 \%$ de las señoras, seguidamente el $21.95 \%$ lo atribuye al "arrepentimiento del esposo", a diferencia del cuadro anterior existe en este grupo un $19.53 \%$ que atribuye el estado post-parto como razón principal para no efectuarse la Cirugía.

Para el grupo de las señoras motivadas en el Centro -Cuadro No. 16- la razón principal para no efectuarse la operación es el "arrepentimiento del esposo" (33.2\%), seguida por el "miedo a la operación" $(29.8 \%)$, sólo $3.2 \%$ cree que la intervención afecta las relaciones sexuales.

Comparando el grupo del ISS con el del Centro, se observa inversión de las razones prioritarias por las cuales las señoras no asistieron a la cita para la intervención quirúrgica.

Para aprovechar al máximo la Encuesta, se agregaron preguntas, completándolas con 
CENTRO

\begin{tabular}{|l|c|r|}
\hline RAZONES & F. & $\%$ \\
\hline $\begin{array}{l}\text { El esposo se } \\
\text { arrepintió }\end{array}$ & 9 & 33.2 \\
\hline $\begin{array}{l}\text { Tuvo miedo } \\
\text { de la operación }\end{array}$ & 7 & 29.8 \\
\hline Otros & 6 & 22 \\
\hline $\begin{array}{l}\text { No tuvo con } \\
\text { quien dejar } \\
\text { los niños }\end{array}$ & 4 & 22 \\
\hline $\begin{array}{l}\text { Cree que la } \\
\text { operación afec- } \\
\text { ta las relaciones } \\
\text { sexuales }\end{array}$ & 1 & 3.2 \\
\hline TOTALES & 27 & \\
\hline
\end{tabular}

CUADRO No. 16

miras a obtener resultados que permitan el mejoramiento de los Programas Institucionales, de acuerdo al concepto que como Institución Educativa tuviese la Entidad en el grupo materia de estudio.

Se transcribe a continuación los resultados de ellas:

- Temor por el método en cuanto a la técnica "son ligadas, no cortadas".

\section{- Trae consecuencias negativas}

- Edificio en construcción (razón temporal)

\section{. Quedan embarazadas de nuevo}

- Impresión que les causa el estado postoperatorio de las otras pacientes, mientras llega el turno que les corresponde.

. Desea tener más hijos.

- La intervención requiere mucho tiempo de convalecencia (?)
Por comentarios de las señoras entrevistadas anteriormente consideran "antihigiénico" permanecer dos en una misma cama mientras se recuperan.

\section{CONCLUSIONES}

De acuerdo con el análisis anterior, concluímos:

1.- Del grupo total de estudio, surgen 2 subgrupos así:

a. Señoras motivadas en el Centro de Profamilia (30)

b) Señoras motivadas en el ISS (46)

Cada grupo con sus correspondientes características de acuerdo al análisis de los datos.

2.- Como promedios generales del grupo, se obtuvieron:

Edad: 32 años

No. de hijos: 4

Ocupación de las señoras: amas de casa

Ocupación del esposo: Obreros

Ingresos Mensuales: $\$ 2.000-3.000$

3.- No se tiene un concepto claro sobre lo que es "Planificación Familiar", para la mayoría del grupo (58\%) es "Evitar la Familia", proviniendo este de los Centros de Salud, seguidamente del ISS e inclusive del "mismo Centro de Profamilia. El método más conocido resultó ser el de "Las Pastas". Aparece además un porcentaje significativo: $39 \%$ con un desconocimiento total sobre métodos.

4.- La Sala de Conferencias es el sitio del Centro donde las señoras reciben la primera información, en el cual se les enseña todos los métodos, sin embargo el de mayor captación es nuevamente el de las Pastas, añadiendo el de Ciru- 
gía como método nuevo. Se observa que solicitan más información en un sentido de reafirmación en cuanto a la efectividad del método escogido, particularmente del de Cirugía.

5.- La aceptación del programa de Cirugía es buená, la mayor parte de las señoras acusan un concepto positivo al respecto. El requisito exigido que obtuvo el mayor porcentaje fue "La autorización firmada por el conyuge" el cual se puede asegurar ha sido uno de los obstáculos que más ha influído en la inasistencia a la intervención, añadiendo a este el "Miedo a la Operación" debido de una parte al número de comunicaciones contradictorias provenientes de personas a las cuales no ha llegado el mensaje de fuentes fidedignas $y$ de otra parte a la insuficiente información y deficiente motivación impartida por el personal que compone los diferentes Estamentos de la Institución.

\section{RECOMENDACIONES}

1.- Capacitación a un nivel más profundo y en términos médicos sobre las diferentes técnicas de Esterilización para el grupo de Motivadoras, con el fin de lograr más efectividad en la transmisión del mensaje.

2.- Revisar los requisitos que actualmente se les exige a las señoras, para fijar y concretar nuevos criterios al respecto.

3.- Delimitar concretamente las funciones de cada una de las Instructoras de Centro, y delegar en una sola persona las concernientes al Programa Quirúrgico con el fin de prestarle una mayor atención al mismo.

4.- Reuniones periódicas de los diferentes Deptos (Educativo, Consultorios, Cirugía) para comentar las anomalías que se puedan presentar en el ejercicio de las funciones de cada uno, con el propósito de modificar la actitud hacia el trabajo en general y el Pro- grama Quirúrgico en particular, obteniendo de este modo una mejor coordinación entre todo el personal de la Institución.

5.- Revisión de las salas de recuperación con el fin de lograr un mejoramiento en los servicios Pre y Post-Operatorio.

\section{RESUMEN}

La disminución en el número de aceptantes de la esterilización llevó a encuestar un grupo de quienes se habían retractado de la intervención y otro de quienes se esperaba fueran sus usuarias a fin de conocer los factores causales.

La autorización firmada por el cónyuge aparece como el obstáculo sobresaliente, junto al "miedo a la operación".

La motivación e información defectuosas se identifican como problemas básicos.

Se investigaron, además, aspectos socioeconómicos, actitudes hacia la Planificación Familiar y la imagen que proyecta Profamilia como Centro Educativo.

\section{SUMMARY}

The diminishing number on the sterilization welcomers carried an investigation on the retracted parts and whom they were expected to be the users in order to know the causing factors. The most outstanding factors were the spouse's signed permit and "fear to the operation".

Defectuous motivation and information are identified with the basic problems.

Socioeconomicalaspects were also studied and the attitudes towards "Planificacion Familiar" too. The image proyected by "Profamilia" as an educational center was analized. 


\section{BIBLIOGRAFIA}

1.- John W. Best. Cómo Investigar en Educación. Edicicnes Norata, Madrid 1965
2.- Murray R. Spiegel, Ph. D. Estadística. Libros Mc.Graw-Hill, 1961.

3.- Folletos. Biblioteca Profamilia Cali. 